\title{
A Novel Active Pining Control for Synchronization of Uncertain Lü Chaotic Systems
}

\author{
Shaocheng Qu Meijing Gong Xiaoyan Wang Hao Qu \\ Department of Information and Technology, Huazhong Normal University, Wuhan 430079, P. R. China
}

\begin{abstract}
This paper discusses the synchronization of uncertain Lü chaotic systems. Based on the idea of active control, a novel active pining control strategy is presented, which only needs a state of uncertain Lü chaotic systems. The proposed controller can achieve synchronization between a salve system and a master system, and ensure the synchronized robust stability of uncertain Lü chaotic systems. Numerical simulations of Lü chaotic systems show that the controller can make chaotic systems achieve synchronization in a quite short period and the synchronization is of good robust stability.
\end{abstract}

Keywords: Lü chaotic system, Pining control, Active control, Synchronize.

\section{Introduction}

Chaos is an interesting phenomenon of nonlinear systems. A deterministic chaotic system has some remarkable dynamic characteristics [1], such as system evolution sensitive to the change in initial conditions and broad spectrum of Fourier transform. It can be treated as a carrier to modulate signals that have the random characteristics. It also has the overall stability. When we use a chaotic signal to drive two identical systems, the two systems or certain parts of them will have the synchronous behavior, which does well for confidential communication.

Chaos synchronization has attracted a great deal of attention since Pecora and Carroll established a chaos synchronization scheme for two identical chaotic systems with different initial conditions. Various effective methods have been presented to synchronize various chaotic systems, for example, Lyapunov method, linear and nonlinear feedback control, adaptive control, impulse control, and so on [2]-[4]. Bai. E. W proposed an active control method to synchronize two Lorenz systems [5]. Song designed time-delayed repetitive learning control to synchronize chaotic systems [6]. Wang studied the stabilization of Chen chaotic system and obtained a unified control strategy [7]. Chen Zhi-sheng used nonlinear feedback control to achieve the synchronization of Lü system [8].

In this paper, the synchronization problem of two uncertain chaotic systems is considered. Based on the idea of active control and the theory of pining control, an active pining controller is designed. The controller achieves synchronization of two chaotic systems with parametric uncertainty and ensures the robust stability of uncertain Lü chaotic system synchronization. The theoretical analysis and numerical simulations are presented to illustrate the proposed controller. The results indicate the controller could synchronize uncertain chaotic systems in any initial condition in a short period, with excellent robust stability.

\section{Problem formulation and active pining control method}

We consider the following two identical nonlinear uncertain chaotic systems. The master system is given by

$$
\dot{x}(t)=(A+\Delta A) x(t)+f(x)
$$

and the salve system is given by

$$
\dot{y}(t)=(A+\Delta A) y(t)+f(y)+u(t)
$$

where $x, y \in R^{n}$ is the state, $u \in R^{m}$ is the control input, the nonlinear function $f$ is continuously differentiable and satisfies the global Lipschitz condition. $A$ is a constant matrix with appropriate dimension, $\Delta A(t)$ denotes parametric uncertainty or structural variation of the system and satisfies $\|\Delta A(t)\| \leq \delta(t)$.

Our goal is to design a simple appropriate controller $u(t)$ such that the trajectory of the salve system (2) asymptotically approaches the master system (1) and achieve synchronization finally.

Defined error $e=y-x$, let (2) subtract from (1), we obtain

$$
\dot{e}(t)=(A+\Delta A) e(t)+F(x, y)+u(t)
$$

where $F(x, y)=f(y)-f(x)$.

According to the idea of active control [5], we can use the control input $u(t)$ to eliminate all items that 
cannot be shown in the form of the error $e$. By this way, the control input can be determined

$$
u(t)=W(t)-F(x, y)
$$

Substituting (4) into (3), equation (3) can be rewritten as

$$
\dot{e}(t)=(A+\Delta A) e(t)+W(t)
$$

Equation (5) describes the error dynamics of synchronization for uncertain chaotic system, and can be considered a common control problem.

Usually, it is difficult to obtain all states for the practical system. So the controller only using partial system state is useful for the synchronization of uncertain Lü chaotic systems.

The pining control only includes a system state, which can stabilize the total system by the coupling of system [9]. As long as controller stabilizes the system (5), the error $e$ will converge to zero in infinite time. This implies that the master system (1) and the salve system (2) are synchronized finally.

\section{A active pining controller for $L \ddot{u}$ chaotic systems}

We consider the following uncertain Lü chaotic systems. The master system is given by

$$
\left\{\begin{array}{l}
\dot{x}_{1}=a\left(x_{2}-x_{1}\right) \\
\dot{x}_{2}=-x_{1} x_{3}+c x_{2}+\Delta d x_{3} \\
\dot{x}_{3}=x_{1} x_{2}-(b+\Delta b) x_{3}
\end{array}\right.
$$

and the salve system is given by

$$
\left\{\begin{array}{l}
\dot{y}_{1}=a\left(y_{2}-y_{1}\right)+u_{1}(t) \\
\dot{y}_{2}=-y_{1} y_{3}+c y_{2}+\Delta d y_{3}+u_{2}(t) \\
\dot{y}_{3}=y_{1} y_{2}-(b+\Delta b) y_{3}+u_{3}(t)
\end{array}\right.
$$

where $\Delta d=k_{1} \varepsilon(t), \Delta b=k_{2} \varepsilon(t)$ are uncertainties, and $k_{1}=0.05, k_{2}=0.1$ are the amplitude values of the stochastic noise $\varepsilon(t)$.

According to the above discussion, the errors dynamic system (5) can be gotten easily. We can obtain the following conclusion.

Theorem: For uncertain Lü chaotic systems (5) with control $W=\left[\begin{array}{lll}0 & g e_{2} & 0\end{array}\right]$, ie. $w_{1}(t)=w_{3}(t)=0$, $w_{2}(t)=g e_{2}$, where the feedback gain

$$
g<-c+\min \left\{-\frac{a}{4 q}, \frac{a\left(b+k_{2}\right)+q k_{1}^{2}}{4 q\left(b-k_{2}\right) q}\right\}
$$

the salve system (7) and the master system (6) are synchronized finally.

Proof: According to the above discussion, errors dynamics system (5) is

$$
\left[\begin{array}{l}
\dot{e}_{1} \\
\dot{e}_{2} \\
\dot{e}_{3}
\end{array}\right]=\left[\begin{array}{ccc}
-a & a & 0 \\
0 & c & k_{1} \varepsilon(t) \\
0 & 0 & -b-k_{2} \varepsilon(t)
\end{array}\right]\left[\begin{array}{l}
e_{1} \\
e_{2} \\
e_{3}
\end{array}\right]+\left[\begin{array}{c}
0 \\
g e_{2} \\
0
\end{array}\right]
$$

ie.

$$
\left\{\begin{array}{l}
\dot{e}_{1}=a\left(e_{2}-e_{1}\right) \\
\dot{e}_{2}=(c+g) e_{2}+k_{1} \varepsilon(t) e_{3} \\
\dot{e}_{3}=-\left(b+k_{2} \varepsilon(t)\right) e_{3}
\end{array}\right.
$$

Consider the Lyapunov function candidate

$$
V=\frac{1}{2} e_{1}^{2}+\frac{q}{2}\left(e_{2}^{2}+e_{3}^{2}\right)
$$

where $q$ is positive constant. The derivative of the Lyapunov function respect to time is

$$
\begin{aligned}
\dot{V} & =e_{1} \cdot \dot{e}_{1}+q\left(e_{2} \cdot \dot{e}_{2}+e_{3} \cdot \dot{e}_{3}\right) \\
& =e_{1}\left[a\left(e_{2}-e_{1}\right)\right]+q\left\{e_{2}\left[(c+g) e_{2}+k_{1} \varepsilon(t) e_{3}\right]-e_{3}\left(b+k_{2} \varepsilon(t)\right) e_{3}\right\} \\
& =-a e_{1}^{2}+a e_{1} e_{2}+q\left[(c+g) e_{2}^{2}+k_{1} \varepsilon(t) e_{2} e_{3}-\left(b+k_{2} \varepsilon(t)\right) e_{3}^{2}\right] \\
& \leq-a e_{1}^{2}+a\left|e_{1}\right|\left|e_{2}\right|+q\left[(c+g) e_{2}^{2}-\left(b+k_{2} \varepsilon(t)\right) e_{3}^{2}+k_{1} \varepsilon(t)\left|e_{2}\right|\left|e_{3}\right|\right] \\
& =-\left(\left|e_{1}\right|,\left|e_{2}\right|,\left|e_{3}\right|\right) P\left(\left|e_{1}\right|,\left|e_{2}\right|,\left|e_{3}\right|\right)^{T}
\end{aligned}
$$

where

$$
P=\left[\begin{array}{ccc}
a & -\frac{1}{2} a & 0 \\
-\frac{1}{2} a & -q(c+g) & -\frac{1}{2} q k_{1} \varepsilon(t) \\
0 & -\frac{1}{2} q k_{1} \varepsilon(t) & q\left(b+k_{2} \varepsilon(t)\right)
\end{array}\right]
$$

If $P>0$, then $\dot{V}<0$ according to (9) and (10). Obviously, matrix inequality $P>0$ is equivalent to the following two inequalities

$$
\begin{gathered}
-a q(c+g)-\frac{1}{4} a^{2}>0 \\
-a q\left(b+k_{2} \varepsilon(t)\right)(c+g)-\frac{a^{2}}{4}\left(b+k_{2} \varepsilon(t)\right)-\frac{a q}{4}\left(k_{1} \varepsilon(t)\right)^{2}>0
\end{gathered}
$$

Solving the above two inequalities, and considering $k_{1}=0.05, k_{2}=0.1$ and $\varepsilon(t) \in\left[\begin{array}{ll}-1 & 1\end{array}\right]$, we can obtain

$$
g<-c+\min \left\{-\frac{a}{4 q}, \frac{a\left(b+k_{2}\right)+q k_{1}^{2}}{4 q\left(b-k_{2}\right) q}\right\}
$$

The proof is completed.

\section{Numerical simulation}

Lü’s chaotic system is given by

$$
\left\{\begin{array}{l}
\dot{x}_{1}=a\left(x_{2}-x_{1}\right) \\
\dot{x}_{2}=-x_{1} x_{3}+c x_{2} \\
\dot{x}_{3}=x_{1} x_{2}-b x_{3}
\end{array}\right.
$$

Where $a, b$ and $c$ are three real positive parameters. When $a=36, b=3$ and $c=20$, Lü's system will behave chaotically in Fig.1. 


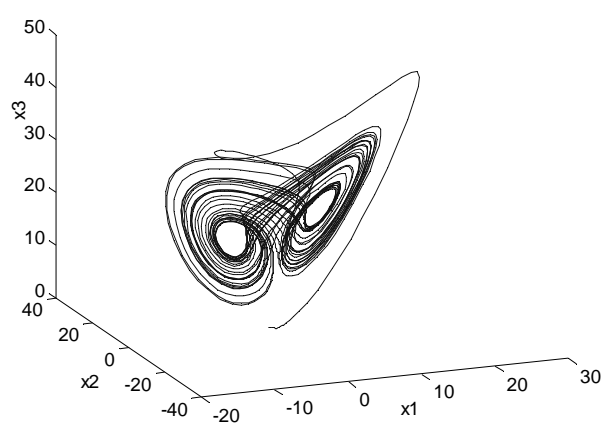

Fig.1. Phase portrait of Lü’s chaotic system.

For uncertain Lü chaotic system (6) and (7), the error dynamics is

$$
\dot{e}=(A+\Delta A) e+F(x, y)+u(t)
$$

where $e=\left(e_{1}, e_{2}, e_{3}\right)^{T}, F(x, y)=f(y)-f(x)$

$u(t)=\left[u_{1}(t), u_{2}(t), u_{3}(t)\right]^{T}$,

$A=\left[\begin{array}{ccc}-a & a & 0 \\ 0 & c & 0 \\ 0 & 0 & -b\end{array}\right], \Delta A=\left[\begin{array}{ccc}0 & 0 & 0 \\ 0 & 0 & k_{1} \varepsilon(t) \\ 0 & 0 & -k_{2} \varepsilon(t)\end{array}\right]$,

$f(x)=\left[\begin{array}{l}0 \\ -x_{1} x_{3} \\ x_{1} x_{2}\end{array}\right], f(y)=\left[\begin{array}{l}0 \\ -y_{1} y_{2} \\ y_{1} y_{2}\end{array}\right]$,

$F(x, y)=\left[\begin{array}{l}0 \\ -y_{1} y_{3}+x_{1} x_{3} \\ y_{1} y_{2}-x_{1} x_{2}\end{array}\right]$,

Defined active control $u(t)=W(t)-F(x, y)$ anc choose the controller as follow

$$
W=\left[\begin{array}{lll}
0 & g e_{2} & 0
\end{array}\right]
$$

where control parameter $g=-60$ is chosen according to the above theory.

Let the initial conditions be $x(0)=\left[\begin{array}{lll}1 & 2 & 0\end{array}\right]^{T}$, $y(0)=\left[\begin{array}{lll}0 & 2 & 1\end{array}\right]^{T}$. We can observe the following numerical results. In 20s, the motion trajectories have entered into the chaotic attractor. From then on, the proposed active pining controller is activated. The numerical results are described in Fig. 2. As we expected, one can observe that the trajectories of the slave system asymptotically approach the ones of the master system. Errors of synchronization of two uncertain chaotic are displayed in Fig. 3. This indicates that uncertain chaotic systems are synchronized and robust stable in the presence of parametric uncertainties finally.
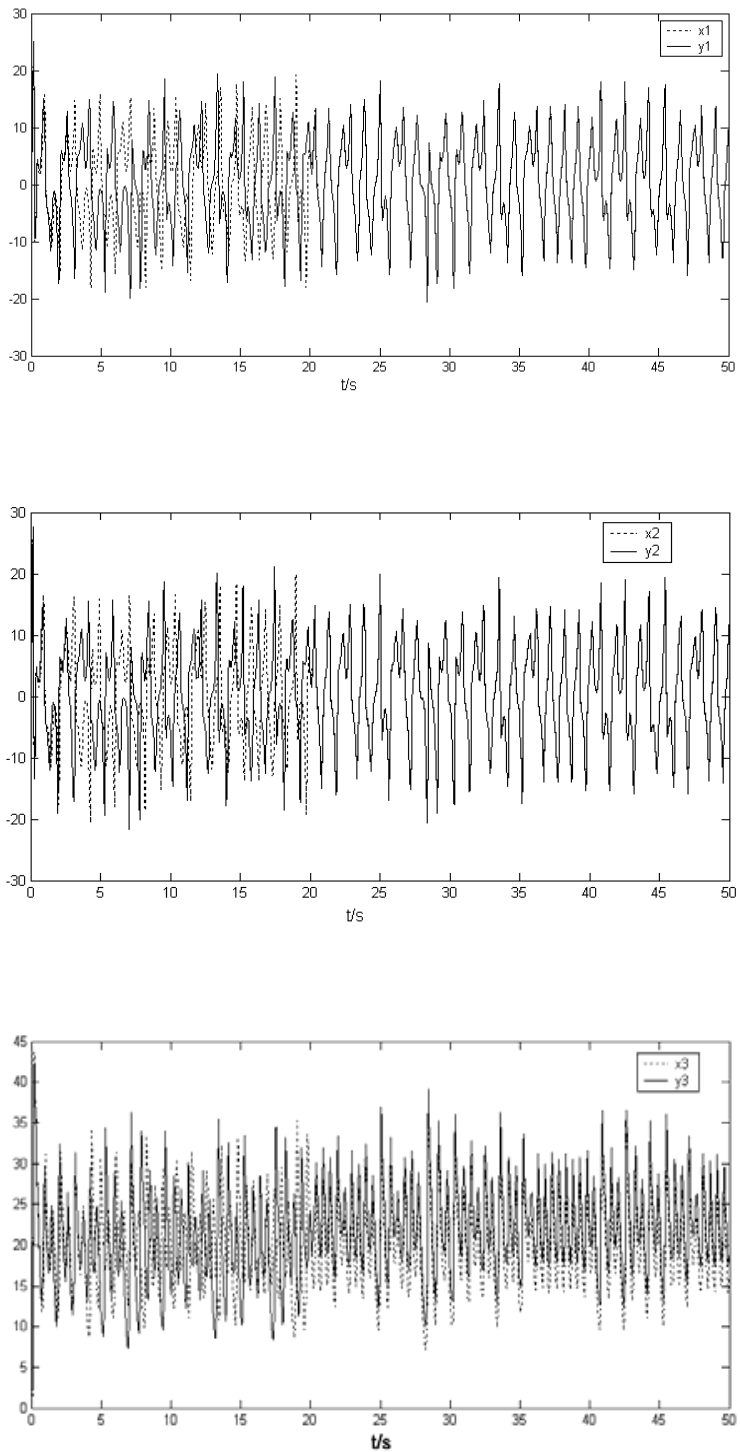

Fig. 2: Synchronization of two Lü’s chaotic system with parametric uncertainties

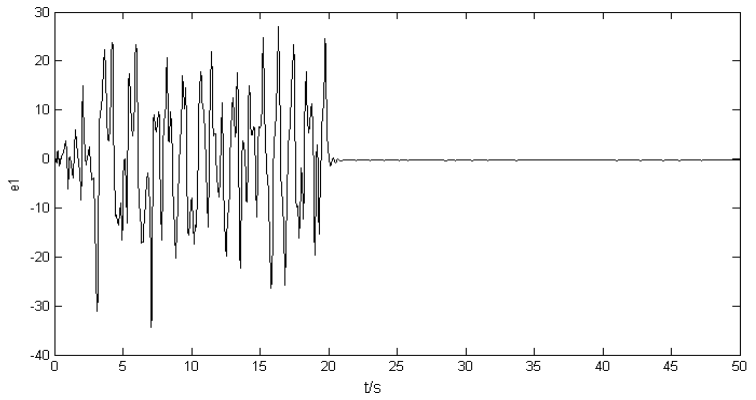



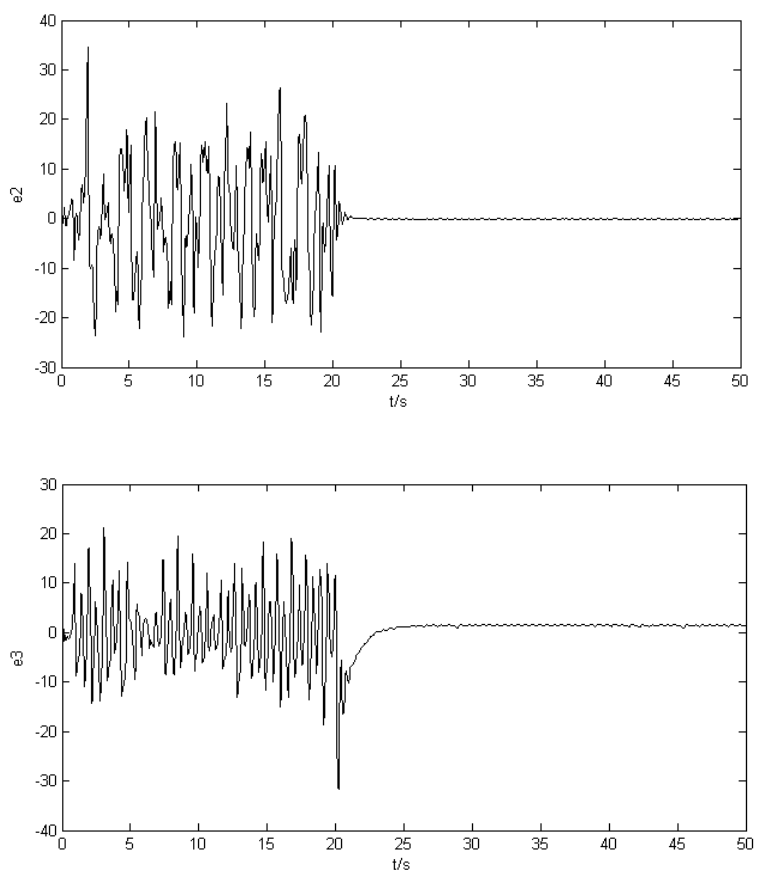

Fig. 3: Error of synchronization of two Lü’s chaotic system with parametric uncertainties.

\section{Conclusions}

In this work, a novel active pining controller was developed for synchronization of uncertain chaotic system. Based on the active control method, the active pining control strategy has been achieved. A simple sufficient condition is drawn for the robust stability of the error dynamics based on Lyapunov stability theory. As the simulations show, the new controller could achieve synchronization of two uncertain chaotic systems and maintain robust stable in the presence of parametric uncertainties.

\section{Acknowledgement}

This work is partially supported by National Nature Science Foundation of China (Grant No. 60274020) and postdoctoral Foundation of Huazhong University of science and technology and "Dangui" Foundation of Huazhong Normal University.

\section{References}

[1] E. Ott, C. Grebogi and J. A. Yorke, Controlling chaos. Phys Rev Lett; 64:1196-1199, 1990.

[2] S.H. Chen, F. Wang and C.P. Wang, Synchronizing strict-feedback chaotic systems via a single controller. Chaos, Solitons and Fractals, 20:235-243, 2004.
[3] M.Y. Chen and Z.Z. Han, Controlling Synchronizing Chaotic Genesio system via nonlinear feedback control. Chaos, Solitons and Fractals, 17: 709-716, 2003.

[4] Y.W. Wang, Z.H. Hong Guan and X.J. Wen, Adaptive synchronization for Chen chaotic system with fully unknown parameters. Chaos, Solitons and Fractals, 19: 899-903, 2004.

[5] E. W. Bai and K E.Lonngren, Synchronization and control chaotic system using active control. Chaos, Solitons and Fractals, 11:39-47, 2000.

[6] Y.X. Song, X.H. Yu and G.R. Chen. Timedelayed repetitive learning control for chaotic systems. Int. J. Bifurcat. Chaos, Solitons and Fractals, 12: 1057-1065, 2002.

[7] J.G. Wang and Zhao-yi, Synchronization of Chen System and a Class of Unified Chaotic System. Circuits and Systems Journal, 12:57-60 2004.

[8] Z.S. Chen, K.H. Sun and T.S. Zhang. A nonlinear feedback synchronization control theorem for Lü chaotic systems Phys Lett A, 54: 2580-2583, 2005.

[9] X.F. Wang, X. Li and G.R. Chen, Complex network theorem and its application. Tsinghua University Press. 2006. 